\title{
Efficient Sialylation with Phenyltrifluoroacetimidates as Leaving Groups
}

\section{Sutang Cai and Biao Yu*}

State Key Laboratory of Bio-organic and Natural Products Chemistry, Shanghai Institute of Organic Chemistry, Chinese Academy of Sciences, Shanghai 200032, China. Email: byu@mail.sioc.ac.cn

\section{Supporting Information}

\section{Preparation of trifluoroacetimidates 2}

To a solution of methyl 5-acetamido-4,7,8,9-tetra- $O$-acetyl-3,5-dideoxy- $\beta$-D-glycero-Dgalacto-2-nonulopyranosonate 1 (1 eq) in acetone was added $\mathrm{K}_{2} \mathrm{CO}_{3}(3$ eq) and $\mathrm{N}$-phenyl trifluoroacetimidoyl chloride (10 eq). After stirring at room temperature for $3 \mathrm{~h}$, the mixture was filtered and then concentrated. The residue was subjected to chromatography on a silica gel column (petroleum ether-EtOAc, $2: 3$ ). Imidate $\mathbf{2 a}(\alpha: \beta=1: 1)$ was thus obtained in $82 \%$ yield. Imidate $\mathbf{2 b}$ ( $\beta$ only) was obtained in $77 \%$ yield.

1.1 Methyl 5-acetamido-4,7,8,9-tetra- $O$-acetyl-3,5-dideoxy- $\beta$-D-glycero-D-galacto-2nonulopyranosonate 2-( $N$-phenyl)trifluoroacetimidate (2a) $\alpha$ Anomer: $[\alpha]_{\mathrm{D}}{ }^{20}=+18.5\left(c 0.95, \mathrm{CHCl}_{3}\right) .{ }^{1} \mathrm{H} \mathrm{NMR}\left(300 \mathrm{MHz}, \mathrm{CDCl}_{3}\right): \delta 7.30-6.70(5 \mathrm{H}, \mathrm{m}$, ArH), 5.42 (1 H, d, $J=9.9 \mathrm{~Hz}, \mathrm{~N}-\mathrm{H}), 5.37$ (1 H, dd, $J=2.4,6.6 \mathrm{~Hz}, \mathrm{H}-7), 5.24$ (1 H, dt, $J=3.0$, $6.3 \mathrm{~Hz}, \mathrm{H}-8), 5.12$ (1 H, dt, $J=5.1,10.2 \mathrm{~Hz}, \mathrm{H}-4), 4.68$ (1 H, dd, $J=2.4,10.8 \mathrm{~Hz}, \mathrm{H}-6), 4.38$ (1 $\mathrm{H}, \mathrm{dd}, J=2.7,12.1 \mathrm{~Hz}, \mathrm{H}-9 \mathrm{a}), 4.23$ (1 H, dd, $J=6.0,12.1 \mathrm{~Hz}, \mathrm{H}-9 \mathrm{~b}), 4.15(1 \mathrm{H}, \mathrm{q}, J=10.5 \mathrm{~Hz}$, H-5), 3.82 (3 H, s, $\left.\mathrm{COOCH}_{3}\right), 2.74(1 \mathrm{H}, \mathrm{dd}, J=5.4,13.2 \mathrm{~Hz}, \mathrm{H}-3 \mathrm{eq}), 2.33$ (1 H, dd, $J=10.5$, $13.5 \mathrm{~Hz}, \mathrm{H}-3 \mathrm{ax}), 2.17,2.05,1.99,1.98,1.91$ (3 H each, s each, OAc and NAc). ${ }^{13} \mathrm{C}$ NMR (75 $\left.\mathrm{MHz}, \mathrm{CDCl}_{3}\right): \delta 170.8,170.5,170.3,170.2,170.0,167.4,142.6,128.7,124.6,119.3,97.9,74.0$, 70.4, 68.4, 67.6, 61.9, 52.9, 49.3, 36.5, 23.1, 20.8, 20.7, 20.6. ESI-MS (m/z): 685.1 (M+ $\left.\mathrm{Na}^{+}\right)$. Anal. Calcd for $\mathrm{C}_{28} \mathrm{H}_{33} \mathrm{~N}_{2} \mathrm{O}_{13} \mathrm{~F}_{3} \cdot 0.5 \mathrm{H}_{2} \mathrm{O}: \mathrm{C}, 50.08 ; \mathrm{H}, 5.10, \mathrm{~N}, 4.17$. Found: C, 50.17; H, 5.10; N, 3.92 .

$\beta$ Anomer: $[\alpha]_{\mathrm{D}}{ }^{20}=-11.1\left(c 1.0, \mathrm{CHCl}_{3}\right) .{ }^{1} \mathrm{H} \mathrm{NMR}\left(300 \mathrm{MHz}, \mathrm{CDCl}_{3}\right): \delta 7.30-6.70(5 \mathrm{H}, \mathrm{m}, \mathrm{ArH})$, $5.43(1 \mathrm{H}, \mathrm{dd}, J=2.4,4.5 \mathrm{~Hz}, \mathrm{H}-7), 5.42(1 \mathrm{H}, \mathrm{d}, J=9.0 \mathrm{~Hz}, \mathrm{NH}), 5.26(1 \mathrm{H}, \mathrm{td}, J=4.8,11.4 \mathrm{~Hz}$, H-4), 5.13 (1 H, m, H-8), 4.56 (1 H, dd, $J=2.4,12.6$ Hz, H-9a), 4.28 (1 H, q, $J=10.8 \mathrm{~Hz}, \mathrm{H}-5)$, 4.20 ( $1 \mathrm{H}, \mathrm{dd}, J=2.4,10.8 \mathrm{~Hz}, \mathrm{H}-6), 4.08$ (1 H, dd, $J=7.2,12.0 \mathrm{~Hz}, \mathrm{H}-9 \mathrm{~b}), 3.82(3 \mathrm{H}, \mathrm{s}$, $\left.\mathrm{COOCH}_{3}\right), 2.80(1 \mathrm{H}, \mathrm{dd}, J=5.1,13.5 \mathrm{~Hz}, \mathrm{H}-3 \mathrm{eq}), 2.16(1 \mathrm{H}, \mathrm{dd}, J=12.0,13.2 \mathrm{~Hz}, \mathrm{H}-3 \mathrm{ax})$, 
2.15, 2.08, 2.06, 1.90, 1.80 (3 H each, s each, OAc and NAc). ${ }^{13} \mathrm{C}$ NMR $\left(75 \mathrm{MHz}, \mathrm{CDCl}_{3}\right): \delta$ 170.9, 170.6, 170.4, 170.2, 170.1, 165.3, 142.6, 128.8, 124.6, 119.0, 99.8, 73.7, 71.7, 68.3, 68.1, 62.4, 53.1, 48.8, 35.6, 23.1, 20.8, 20.7, 20.4. ESI-MS ( $m / z): 680.1\left(\mathrm{M}+\mathrm{NH}_{4}^{+}\right), 685.1\left(\mathrm{M}+\mathrm{Na}^{+}\right)$. Anal. Calcd for $\mathrm{C}_{28} \mathrm{H}_{33} \mathrm{~N}_{2} \mathrm{O}_{13} \mathrm{~F}_{3} \cdot \mathrm{H}_{2} \mathrm{O}: \mathrm{C}, 49.42 ; \mathrm{H}, 5.18 ; \mathrm{N}$, 4.12; Found: C, 49.56; H, 5.07; N, 3.98 .

1.2 Methyl 5-acetamido-4,7,8,9-tetra- $O$-acetyl-3,5-dideoxy- $\beta$-D-glycero-D-galacto-2nonulopyranosonate 2-(N-p-nitrophenyl)trifluoroacetimidate (2b) $\beta$ Anomer: $[\alpha]_{\mathrm{D}}{ }^{20}=-46.1\left(c 0.89, \mathrm{CHCl}_{3}\right) .{ }^{1} \mathrm{H}$ NMR $\left(300 \mathrm{MHz}, \mathrm{CDCl}_{3}\right): \delta 8.19(2 \mathrm{H}, \mathrm{d}, J=8.5$ $\mathrm{Hz}, \operatorname{ArH}), 6.90(2 \mathrm{H}, \mathrm{d}, J=8.8 \mathrm{~Hz}, \operatorname{ArH}), 5.57(1 \mathrm{H}, \mathrm{d}, J=4.9 \mathrm{~Hz}), 5.42(1 \mathrm{H}, \mathrm{d}, J=3.8 \mathrm{~Hz})$, $5.28(1 \mathrm{H}, \mathrm{m}), 5.19(1 \mathrm{H}, \mathrm{m}), 4.50(1 \mathrm{H}, \mathrm{d}, J=12.4 \mathrm{~Hz}), 4.24(2 \mathrm{H}, \mathrm{m}), 4.10(1 \mathrm{H}, \mathrm{dd}, J=6.9$, $12.1 \mathrm{~Hz}), 3.84\left(3 \mathrm{H}, \mathrm{s}, \mathrm{COOCH}_{3}\right), 2.84(1 \mathrm{H}, \mathrm{dd}, J=4.7,13.7 \mathrm{~Hz}, \mathrm{H}-3 \mathrm{eq}), 2.20$ (1 H, m, H-3ax), 2.16, 2.09, 2.06, 1.93, 1.83 (3 H each, s each, OAc and NAc). ${ }^{13} \mathrm{C}$ NMR $\left(75 \mathrm{MHz}, \mathrm{CDCl}_{3}\right): \delta$ 170.8, 170.4, 170.4, 170.3, 170.1, 164.9, 148.5, 144.7, 124.8, 119.7, 100.8, 73.7, 70.9, 68.0, 67.9, 62.3, 53.4, 48.9, 35.5, 23.1, 20.8, 20.7, 20.4. ESI-MS $(m / z): 730.0\left(\mathrm{M}+\mathrm{Na}^{+}\right)$. Anal. Calcd for $\mathrm{C}_{28} \mathrm{H}_{32} \mathrm{~N}_{2} \mathrm{O}_{15} \mathrm{~F}_{3} \cdot \mathrm{H}_{2} \mathrm{O}: \mathrm{C}, 46.35 ; \mathrm{H}, 4.72 ; \mathrm{N}, 5.79$; Found: $\mathrm{C}, 46.50 ; \mathrm{H}, 4.34 ; \mathrm{N}, 5.38$.

2. Typical procedure for sialylation with trifluoroacetimidate donors $\mathbf{2 a}$ and $\mathbf{2 b}$

A mixture of the donor $\mathbf{2 a}(80 \mathrm{mg}, 0.12 \mathrm{mmol})$, acceptor HOR 3a-h $(1.5 \mathrm{eq})$, and $3 \AA$ molecular sieves in dry $\mathrm{CH}_{2} \mathrm{Cl}_{2} / \mathrm{CH}_{3} \mathrm{CN}(1: 1,5 \mathrm{~mL})$ was stirred at room temperature under argon for $30 \mathrm{~min}$, and then cooled to $-65^{\circ} \mathrm{C}$. TMSOTf $(0.2 \mathrm{eq})$ was added. After stirring at $-65^{\circ} \mathrm{C}$ for $1.5 \mathrm{~h}$, the mixture was warmed up to room temperature and quenched with a few drops of triethylamine. The resulting mixture was filtered and concentrated. The residue was chromatographed on a silica gel column to afford the desired coupling product $\mathbf{4 a - h}$.

2.1. [Methyl 5-acetamido-4,7,8,9-tetra- $O$-acetyl-3,5-dideoxy- $\beta$-D-glycero- $\alpha / \beta$-D-galacto-2nonulopyranosonate]-(2 $\rightarrow 6$ )-methyl 2,3,4-tri- $O$-benzyl- $\alpha$-D-glucopyranoside (4a) Known compound, see: Sim, M. M.; Kondo, H.; Wong,C.-H. J. Am. Chem. Soc. 1993, 115, 2260.

2.2 [Methyl 5-acetamido-4,7,8,9-tetra- $O$-acetyl-3,5-dideoxy- $\beta$-D-glycero- $\alpha / \beta$-D-galacto-2nonulopyranosonate]-(2 $\rightarrow 6)$-1,2;3,4-di- $O$-isopropylidene- $\alpha$-D-galacopyranoside (4b)

Known compounds, see: Kirchner, E.; Thiem, F.; Dernick, R.; Heukeshoven, J.; Thiem, J. J. 
Carbohydr. Chem. 1988, 7, 453.

2.3 [Methyl 5 -acetamido-4,7,8,9-tetra- $O$-acetyl-3,5-dideoxy- $\beta$-D-glycero- $\alpha / \beta$-D-galacto-2nonulopyranosonate]-(2 $\rightarrow 6)$-1,3,4-tri- $O$-acetyl-2-deoxy-2-phthalimido- $\alpha$-D-glucopyranoside (4c)

${ }^{1} \mathrm{H}$ NMR (300 MHz, $\left.\mathrm{CDCl}_{3}\right): \delta 2.67(0.83 \mathrm{H}, \mathrm{dd}, J=4.2,12.6 \mathrm{~Hz}, \mathrm{H}-3$ eq ( $\alpha$ anomer)), 2.47 (0.21 $\mathrm{H}, \mathrm{dd}, J=4.2,13.5 \mathrm{~Hz}$, H-3eq ( $\beta$ anomer)). HRMS $(m / z)$ : Calcd for $\mathrm{C}_{40} \mathrm{H}_{48} \mathrm{~N}_{2} \mathrm{O}_{22} \mathrm{Na}\left(\mathrm{M}+\mathrm{Na}^{+}\right)$ 931.2591; Found: 931.2608.

2.4 28-O-Benzyl-oleanate-3-yl methyl 5 -acetamido-4,7,8,9-tetra- $O$-acetyl-3,5-dideoxy- $\beta$-Dglycero- $\alpha / \beta$-D-galacto-2-nonulopyranosonate (4d)

$\alpha$ Anomer: $[\alpha]_{\mathrm{D}}{ }^{20}=+24.8\left(c 0.82, \mathrm{CHCl}_{3}\right) .{ }^{1} \mathrm{H}$ NMR $\left(300 \mathrm{MHz}, \mathrm{CDCl}_{3}\right): \delta$ 7.35-7.32 (5 H, $\left.\mathrm{ArH}\right)$, $5.38(1 \mathrm{H}, \mathrm{td}, J=2.4,8.1 \mathrm{~Hz}), 5.30(2 \mathrm{H}, \mathrm{d}, J=6.3 \mathrm{~Hz}), 5.20(1 \mathrm{H}, \mathrm{d}, J=10.2 \mathrm{~Hz}), 5.07(2 \mathrm{H}$, m) , $4.83(1 \mathrm{H}, \mathrm{td}, J=4.5,12.3 \mathrm{~Hz}), 4.40(1 \mathrm{H}, \mathrm{dd}, J=2.4,12.3 \mathrm{~Hz}), 4.09-4.02(2 \mathrm{H}, \mathrm{m}), 3.87(1$ $\mathrm{H}, \mathrm{dd}, J=1.5,11.1 \mathrm{~Hz}), 3.76\left(3 \mathrm{H}, \mathrm{s}, \mathrm{COOCH}_{3}\right), 3.64(1 \mathrm{H}, \mathrm{dd}, J=4.5,11.7 \mathrm{~Hz}), 2.90(1 \mathrm{H}, \mathrm{dd}$, $J=4.2,14.7 \mathrm{~Hz}), 2.65(1 \mathrm{H}, \mathrm{dd}, J=4.8,12.6 \mathrm{~Hz}, \mathrm{H}-3 \mathrm{eq})[\underline{2.54}(1 \mathrm{H}, \mathrm{dd}, J=4.8,12.9 \mathrm{~Hz}, \mathrm{H}-3 \mathrm{eq}$ ( $\beta$ anomer $)$ )], 2.14, 2.11, 2.03, 2.03, 1.89 (3 H each, s each, OAc and NAc), 1.16, 0.98, 0.91, 0.89, 0.86, 0.75, 0.60 (3 H each, $\left.\mathrm{CH}_{3}\right) .{ }^{13} \mathrm{C} \mathrm{NMR}\left(75 \mathrm{MHz}, \mathrm{CDCl}_{3}\right): \delta 177.5,171.0,170.5,170.3$, $170.1,169.8,169.0,143.8,136.4,128.4,128.0,127.9,125.5,122.5,97.0,80.3,72.2,69.3,69.1$, $67.6,65.9,62.5,55.2,52.4,49.7,47.2,46.8,45.9,41.7,41.4,39.3,38.5,38.3,38.2,36.6,33.9$, 33.1, 32.7, 32.4, 30.7, 29.7, 28.1, 27.7, 25.9, 24.2, 23.6, 23.4, 23.2, 21.1, 20.8, 20.75, 18.4, 16.9, 16.5, 15.3. HRMS (m/z): Calcd for $\mathrm{C}_{57} \mathrm{H}_{81} \mathrm{NO}_{15} \mathrm{Na}\left(\mathrm{M}+\mathrm{Na}^{+}\right)$1042.5498; Found: 1042.5479 .

2.5 Diosgenin-3-yl methyl 5-acetamido-4,7,8,9-tetra- $O$-acetyl-3,5-dideoxy- $\beta$-D-glycero- $\alpha / \beta$-Dgalacto-2-nonulopyranosonate (4e)

$\alpha$ Anomer: $[\alpha]_{\mathrm{D}}{ }^{20}=-57.0\left(c 0.70, \mathrm{CHCl}_{3}\right) .{ }^{1} \mathrm{H}$ NMR $\left(300 \mathrm{MHz}, \mathrm{CDCl}_{3}\right): \delta$ 5.38-5.28 $(2 \mathrm{H}, \mathrm{m})$, $5.18(1 \mathrm{H}, \mathrm{br}), 4.85(1 \mathrm{H}, \mathrm{br}), 4.41(1 \mathrm{H}, \mathrm{q}, J=8.1 \mathrm{~Hz}), 4.34(1 \mathrm{H}, \mathrm{dd}, J=2.1,12.6 \mathrm{~Hz}), 4.15(1$ $\mathrm{H}, \mathrm{dd}, J=5.1,12.3 \mathrm{~Hz}), 4.04(2 \mathrm{H}, \mathrm{m}), 3.79\left(3 \mathrm{H}, \mathrm{s}, \mathrm{COOCH}_{3}\right), 3.67-3.59(1 \mathrm{H}, \mathrm{m}), 3.45(1 \mathrm{H}$, dd, $J=6.0,9.3 \mathrm{~Hz}), 3.37(1 \mathrm{H}, \mathrm{t}, J=10.8 \mathrm{~Hz}), 2.59(1 \mathrm{H}, \mathrm{dd}, J=4.5,12.9 \mathrm{~Hz}, \mathrm{H}-3 \mathrm{eq})[2.51(1 \mathrm{H}$, dd, $J=4.5,13.2 \mathrm{~Hz}, \mathrm{H}-3 \mathrm{eq}(\beta$ anomer $))], 2.41-2.29(2 \mathrm{H}, \mathrm{m}), 2.15,2.13,2.03,2.03,1.88(3 \mathrm{H}$ 
each, s each, OAc and NAc), 1.00, 0.78 (3 $\mathrm{H}$ each, s each, $\left.\mathrm{CH}_{3}\right), 0.97(3 \mathrm{H}, \mathrm{d}, J=6.9 \mathrm{~Hz}), 0.79$ $(3 \mathrm{H}, \mathrm{d}, J=6.0 \mathrm{~Hz}) .{ }^{13} \mathrm{C} \mathrm{NMR}\left(75 \mathrm{MHz}, \mathrm{CDCl}_{3}\right): \delta 171.0,170.5,170.2,170.1,170.0,168.9,140.3$, $121.7,109.2,98.7,80.8,75.3,72.8,69.6,69.2,67.7,66.8,62.2,62.1,56.5,52.6,49.9,49.5,41.6$, 41.0, 40.2, 39.7, 38.3, 37.2, 36.6, 32.1, 31.8, 31.4, 30.3, 29.6, 29.1, 28.8, 23.1, 21.0, 20.8, 19.2, 17.1, 16.2, 14.5. HRMS (m/z): calcd for $\mathrm{C}_{47} \mathrm{H}_{69} \mathrm{NO}_{15} \mathrm{Na}\left(\mathrm{M}+\mathrm{Na}^{+}\right)$910.4560; Found: 910.4570 .

2.6 [Methyl 5-acetamido-4,7,8,9-tetra- $O$-acetyl-3,5-dideoxy- $\beta$-D-glycero- $\alpha / \beta$-D-galacto-2nonulopyranosonate]-(2 $\rightarrow 3$ )-methyl 2-azido-2-deoxy-4,6-O-p-methoxybenzylidene- $\alpha$-Dgalactopyranoside (4f)

$\alpha$ Anomer: $[\alpha]_{\mathrm{D}}{ }^{20}=+60.1\left(c 1.1, \mathrm{CHCl}_{3}\right) .{ }^{1} \mathrm{H} \mathrm{NMR}\left(300 \mathrm{MHz}, \mathrm{CDCl}_{3}\right): \delta 7.43(2 \mathrm{H}, \mathrm{d}, J=9.0 \mathrm{~Hz}$, ArH), 6.89 (2 H, d, $J=8.4 \mathrm{~Hz}, \mathrm{ArH}), 5.41-5.30(2 \mathrm{H}, \mathrm{m}), 5.36(1 \mathrm{H}, \mathrm{s}, \mathrm{PhCH}), 4.87(1 \mathrm{H}, \mathrm{d}, J=$ $3.3 \mathrm{~Hz}), 4.83(1 \mathrm{H}, \mathrm{m}), 4.73(1 \mathrm{H}, \mathrm{dd}, J=3.3,10.5 \mathrm{~Hz}), 4.32(1 \mathrm{H}, \mathrm{dd}, J=2.7,12.6 \mathrm{~Hz}), 4.21-$ $4.17(3 \mathrm{H}, \mathrm{m}), 4.13-4.04(3 \mathrm{H}, \mathrm{m}), 3.97(1 \mathrm{H}, \mathrm{dd}, J=3.9,10.8 \mathrm{~Hz}), 3.81\left(3 \mathrm{H}, \mathrm{s}, \mathrm{COOCH}_{3}\right), 3.69$ $(1 \mathrm{H}, \mathrm{s}), 3.48,3.45\left(3 \mathrm{H}\right.$ each, s each, $\left.\mathrm{OCH}_{3}\right), 2.74(1 \mathrm{H}, \mathrm{dd}, J=4.5,12.6 \mathrm{~Hz}, \mathrm{H}-3 \mathrm{eq})[\underline{2.65}(1 \mathrm{H}$, $\underline{\mathrm{dd}, J}=4.2,12.9 \mathrm{~Hz}, \mathrm{H}-3 \mathrm{eq}(\beta$ anomer $))], 2.16$ (1 H, m, H-3ax), 2.22, 2.17, 2.06, 2.05, $1.90(3 \mathrm{H}$ each, s each, OAc and NAc). ${ }^{13} \mathrm{C}$ NMR (75 MHz, $\left.\mathrm{CDCl}_{3}\right): \delta 170.9,170.5,170.2,170.1,168.6$, $130.4,127.6,113.4,100.5,99.3,97.1,73.2,72.6,70.0,69.1,68.6,68.0,67.1,62.3,58.0,55.5$, 55.3, 52.7, 49.6, 38.4, 23.2, 21.3, 20.8. HRMS $(m / z)$ : Calcd for $\mathrm{C}_{35} \mathrm{H}_{46} \mathrm{~N}_{4} \mathrm{O}_{18} \mathrm{Na}\left(\mathrm{M}+\mathrm{Na}^{+}\right)$: 833.2699; Found: 833.2689.

2.7. [Methyl 5-acetamido-4,7,8,9-tetra- $O$-acetyl-3,5-dideoxy- $\beta$-D-glycero- $\alpha / \beta$-D-galacto-2nonulopyranosonate]-(2 $\rightarrow 3$ )-benzyl 2,6-di-O-benzyl- $\alpha$-D-galactopyranoside $(\mathbf{4 g})$ $\alpha$ Anomer: $[\alpha]_{\mathrm{D}}{ }^{20}=+28.8\left(c 0.76, \mathrm{CHCl}_{3}\right) .{ }^{1} \mathrm{H}$ NMR $\left(300 \mathrm{MHz}, \mathrm{CDCl}_{3}\right): \delta$ 7.45-7.26 $(15 \mathrm{H}, \mathrm{m}$, ArH), $5.28(2 \mathrm{H}, \mathrm{d}, J=1.5 \mathrm{~Hz}), 5.20(1 \mathrm{H}, \mathrm{d}, J=6.0 \mathrm{~Hz}), 4.96-4.90(1 \mathrm{H}, \mathrm{m}), 4.89(1 \mathrm{H}, \mathrm{d}, J=$ $3.6 \mathrm{~Hz}), 4.79-4.46$ (6 H, m), $4.43(1 \mathrm{H}, \mathrm{dd}, J=3.0,10.2 \mathrm{~Hz}), 4.34(1 \mathrm{H}, \mathrm{d}, J=11.7 \mathrm{~Hz}), 4.09-$ $3.96(5 \mathrm{H}, \mathrm{m}), 3.82\left(3 \mathrm{H}, \mathrm{s}, \mathrm{COOCH}_{3}\right), 3.78(1 \mathrm{H}, \mathrm{dd}, J=3.9,9.9 \mathrm{~Hz}), 3.68(1 \mathrm{H}, \mathrm{dd}, J=6.0,9.9$ Hz), 3.00 (1 H, br), 2.53 (1 H, dd, $J=5.4,12.6 \mathrm{~Hz}, \mathrm{H}-3 \mathrm{eq})[\underline{2.46}(1 \mathrm{H}, \mathrm{dd}, J=4.2,13.5 \mathrm{~Hz}, \mathrm{H}-$ 3eq $(\beta$ anomer $))], 2.27$ (1 H, t, $J=12.0 \mathrm{~Hz}, \mathrm{H}-3 \mathrm{ax}), 2.10,2.01,2.00,1.98,1.88$ (3 H each, s each, OAc and NAc). ${ }^{13} \mathrm{C}$ NMR (75 MHz, $\left.\mathrm{CDCl}_{3}\right): \delta 170.8,170.6,170.2,170.1,170.0,168.3,138.5$, $138.2,137.3,128.3,128.2,128.1,127.9,127.7,127.5,127.4,99.3,96.0,74.1,73.4,73.1,73.0$, 
72.8, 69.8, 69.7, 69.2, 69.1, 68.9, 68.7, 67.3, 62.3, 53.0, 49.2, 35.5, 23.1, 21.0, 20.8, 20.6, 20.5. HRMS ( $/ z / z)$ : Calcd for $\mathrm{C}_{47} \mathrm{H}_{57} \mathrm{NO}_{18} \mathrm{Na}\left(\mathrm{M}+\mathrm{Na}^{+}\right)$: 946.3467; Found: 946.3449 .

For the corresponding $\beta$-galactopyranoside analogue \{[Methyl 5-acetamido-4,7,8,9-tetra- $O$ acetyl-3,5-dideoxy- $\beta$-D-glycero- $\alpha / \beta$-D-galacto-2-nonulopyranosonate]-(2 $\rightarrow 3$ )-benzyl $\quad 2,6$-di- $O$ benzyl- $\underline{\beta}$-D-galactopyranoside\}, see: Numata, M.; Sugimoto, M.; Koike, K.; Ogawa, T. Carbohydr. Res. 1987, 163, 209.

2.7. [Methyl 5-acetamido-4,7,8,9-tetra- $O$-acetyl-3,5-dideoxy- $\beta$-D-glycero- $\alpha / \beta$-D-galacto-2nonulopyranosonate]-(2 $\rightarrow 6$ )-methyl 3-O-benzoyl- $\alpha$-D-galactopyranoside (4h) $\alpha$ Anomer: $[\alpha]_{\mathrm{D}}{ }^{20}=+46.5\left(c 1.0, \mathrm{CHCl}_{3}\right) .{ }^{1} \mathrm{H}$ NMR $\left(300 \mathrm{MHz}, \mathrm{CDCl}_{3}\right): \delta 8.15-7.43(5 \mathrm{H}, \mathrm{ArH})$, 5.39-5.32 (2 H, m), $5.26(1 \mathrm{H}, \mathrm{dd}, J=3.0,10.2 \mathrm{~Hz}), 5.23(1 \mathrm{H}, \mathrm{d}, J=8.7 \mathrm{~Hz}), 4.91(1 \mathrm{H}, \mathrm{d}, J=$ $3.6 \mathrm{~Hz}), 4.91-4.86(1 \mathrm{H}, \mathrm{m}), 4.39(1 \mathrm{H}, \mathrm{dd}, J=2.1,12.3 \mathrm{~Hz}), 4.30-4.19(2 \mathrm{H}, \mathrm{m}), 4.09-3.98(4 \mathrm{H}$, m), $3.89(1 \mathrm{H}, \mathrm{dd}, J=5.1,9.3 \mathrm{~Hz}), 3.81\left(3 \mathrm{H}, \mathrm{s}, \mathrm{COOCH}_{3}\right), 3.80-3.74(1 \mathrm{H}, \mathrm{m}), 3.49(3 \mathrm{H}, \mathrm{s}$, $\mathrm{OCH}_{3}$ ), $2.58(1 \mathrm{H}, \mathrm{dd}, J=4.5,12.6 \mathrm{~Hz}, \mathrm{H}-3 \mathrm{eq}), 2.05$ (1 H, overlaped, H-3ax), 2.13, 2.11, 2.03, 1.95, 1.82 (3 H each, s each, OAc and NAc). ${ }^{13} \mathrm{C}$ NMR (75 MHz, $\left.\mathrm{CDCl}_{3}\right): \delta 170.9,170.3,170.2$, 168.1, 166.5, 133.2, 129.9, 128.3, 99.8, 98.7, 74.0, 72.9, 69.3, 69.0, 68.4, 67.7, 67.5, 67.3, 63.0, 62.6, 55.5, 53.0, 49.4, 37.0, 23.1, 21.1, 20.8, 20.7, 20.6. HRMS (m/z): calcd for $\mathrm{C}_{34} \mathrm{H}_{45} \mathrm{NO}_{19} \mathrm{Na}$ $\left(\mathrm{M}+\mathrm{Na}^{+}\right)$: 794.2478; Found: 794.2458.

For the corresponding $\beta$-galactopyranoside analogue \{[Methyl 5-acetamido-4,7,8,9-tetra- $O$ acetyl-3,5-dideoxy- $\beta$-D-glycero- $\alpha / \beta$-D-galacto-2-nonulopyranosonate]-(2 $\rightarrow 6)$-methyl 3-Obenzoyl- $\beta$-D-galactopyranoside\}, see: Marra, A.; Sinay, P. Carbohydr. Res. 1990, 195, 303. 